\title{
Transport in tunneling recombination junctions: A combined computer simulation study
}

\author{
M. Vukadinović, ${ }^{\text {a) }}$ F. Smole, and M. Topič \\ Faculty of Electrical Engineering, University of Ljubljana, Trzaska 25, SI-1000 Ljubljana, Slovenia \\ R. E. I. Schropp \\ Debye Institute (Surfaces, Interfaces and Devices), Utrecht University, P.O. Box 80000 NL-3508 TA \\ Utrecht, The Netherlands \\ F. A. Rubinellib) \\ INTEC, Universidad Nacional del Litoral, Güemes 3450, 3000 Santa Fe, Argentina
}

(Received 12 May 2004; accepted 7 September 2004)

\begin{abstract}
The implementation of trap-assisted tunneling of charge carriers into numerical simulators ASPIN and D-AMPS is briefly described. Important modeling details are highlighted and compared. In spite of the considerable differences in both approaches, the problems encountered and their solutions are surprisingly similar. Simulation results obtained for several tunneling recombination junctions made of amorphous silicon $(a-\mathrm{Si})$, amorphous silicon carbide $(a-\mathrm{SiC})$, or microcrystalline silicon $(\mu c-\mathrm{Si})$ are analyzed. Identical conclusions can be drawn using either of the simulators. Realistic performances of $a-\mathrm{Si} / a$-Si tandem solar cells can be reproduced with simulation programs by assuming that extended-state mobility increases exponentially with the electric field. The same field-enhanced mobilities are needed in single tunneling recombination junctions in order to achieve measured current levels. Temperature dependence of the current-voltage characteristics indicates that the activation energy of enhanced mobilities should be determined. Apparent discrepancies between simulation results and measurements are explained and eliminated making use of Gill's law. For application in tandem and triple solar cell structures, tunneling recombination junctions made of $(\mu c-\mathrm{Si})$ are the most promising of all examined structures. () 2004 American Institute of Physics. [DOI: 10.1063/1.1811375]
\end{abstract}

\section{INTRODUCTION}

Computer-aided design (CAD) of optoelectronic devices is an important tool for testing theories obtained through extensive material research. Simulation programs provide insights into a device's internal and external properties, and give a profound understanding of the relations between relevant device and material parameters. The main advantage of $\mathrm{CAD}$ is the negligible cost of the evaluation of different designs in comparison to pilot production of real devices. However, there is a major drawback-domains, where the assumptions accounted for in the built-in models may no longer be valid due to some other prevailing physical phenomena, are sometimes vaguely defined or not known in advance. Thus, revalidation of models by reproduction of different measured characteristics remains an essential procedure in numerical modeling.

In this paper, the physics controlling the electric transport in amorphous and microcrystalline structures regularly used as tunneling recombination junctions (TRJs) of $a$-Si-based tandem solar cells are explored with two independently developed simulation tools: ASPIN (Ref. 1) and D-AMPS. ${ }^{2,3}$ When standard numerical procedures (well established in the device modeling of amorphous and microcrys-

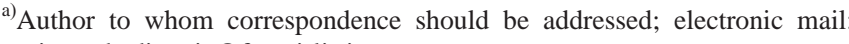
miso.vukadinovic@fe.uni-lj.si

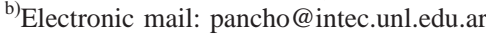

talline structures) were applied, high currents measured in $n-p$ or in $n-p^{+}-p$ junctions ${ }^{4}$ could not be explained. ${ }^{5,6}$ Tunneling of charge carriers via localized energy levels within the semiconductor mobility gap-previously not included in numerical simulators - was believed to be the main contribution to the total current. In addition to the well known PooleFrenkel effect, ${ }^{7}$ three important theories were derived from the same basis by Vincent et al., ${ }^{8}$ Hurkx et al., ${ }^{9}$ and Furlan. ${ }^{10}$ They were successfully integrated into several existing numerical simulators. ${ }^{2,11,12}$ However, we will show, in agreement with the results obtained by Willemen ${ }^{11}$ that in most TRJs it is not possible to reproduce the current levels found experimentally, even when these transport mechanisms are considered.

This paper is organized as follows: in the following section, we briefly describe the numerical simulators ASPIN and D-AMPS with emphasis on the trap-assisted tunneling algorithms implemented in each of the computer codes. In Sec. III, we compare our computer predictions with the results published by Hegedus et al. ${ }^{4}$ Five TRJ structures combining amorphous silicon $(a-\mathrm{Si})$ layers with amorphous silicon carbide $(a-\mathrm{SiC})$ and microcrystalline silicon $(\mu c-\mathrm{Si})$ layers are studied in parallel. In Sec. IV, we discuss the sensitivity of the performance of $a-\mathrm{Si} / a-\mathrm{Si}$ tandem solar cells to the selected TRJ structure. Finally, in Sec. V, we examine how temperature influences TRJ and tandem solar cell performances. Apparently unacceptable discrepancies between ex- 
perimental and simulated results are explained and eliminated by applying Gill's law.

\section{ASPIN AND D-AMPS IN PARALLEL}

\section{A. Brief description of ASPIN's and D-AMPS' computer codes}

Simulation programs require many input parametersmeasured and/or conceptual - in order to produce plausible output results that comply with the presupposed physical laws. Material parameters describe individual layers (layer thickness, energy gap, density of states, etc.) of the structure under study; external parameters determine the working conditions (temperature, illumination, etc.), while system parameters control the execution of the numerical algorithm (simulation type, order of subroutines, precision, etc.). The complex set of semiconductor nonlinear differential equations can be reduced to the modified continuity equations and Poisson's equation (three unknowns per discretization point have to be calculated). ${ }^{1,3,13}$ Many other equations, such as generation-recombination process-related expressions, also have to be included. The entire system has to be selfconsistently solved in accordance with the given boundary conditions by an iterative algorithm.

The numerical algorithm of ASPIN (Ref. 1) is based on finite differences. The system of semiconductor equations is solved with a customized block nonlinear iterative algorithm based on Gummel's method. ${ }^{13}$ Modifications of the original Gummel algorithm were introduced in order to reduce execution time while keeping the same precision and to improve robustness, i.e., to improve convergence. It is beyond the scope of this paper to give more details on these modifications. Some information, e.g., usage of Bernoulli functions, first suggested by Scharfetter and Gummel, can be found in Refs. 13 and 14. The ASPIN's algorithm calculates free-carrier concentrations of holes $(p)$ and electrons $(n)$, and the electrostatic potential $(V)$. The implementation of the generation-recombination process follows the ShockleyRead-Hall (SRH) and Sah-Shockley statistics for continuously distributed density of states (DOS). Traps (allowed energy levels that form DOS) inside the mobility gap can be described by the standard (predefined distribution) or "defect pool" models, as uncorrelated and/or amphoteric states (Gaussians) and exponential tails. Optical properties are calculated with an advanced optoelectronic simulator Sunshine ${ }^{15}$ embedded in ASPIN. Originally, ASPIN was designed for the simulation of single $a$-Si pin solar cells where the consideration of trap-assisted tunneling was not necessary, and therefore no such models were initially developed.

D-AMPS (Ref. 2) is an updated version of the simulation program AMPS. ${ }^{3}$ Like ASPIN, it is based on the method of finite differences. The D stands for developments introduced in the last decade: tunneling through thin barriers, amphoteric states, light scattering on rough surfaces, defect pool model, etc. D-AMPS uses the Newton-Raphson iteration method to solve the Poisson and continuity equations. Both quasi-Fermi levels $\left(E_{F p}\right.$ and $\left.E_{F n}\right)$ and the electron potential $(V)$ were chosen as the three unknowns to be calculated. Describing DOS is similar as in ASPIN. Tunneling through

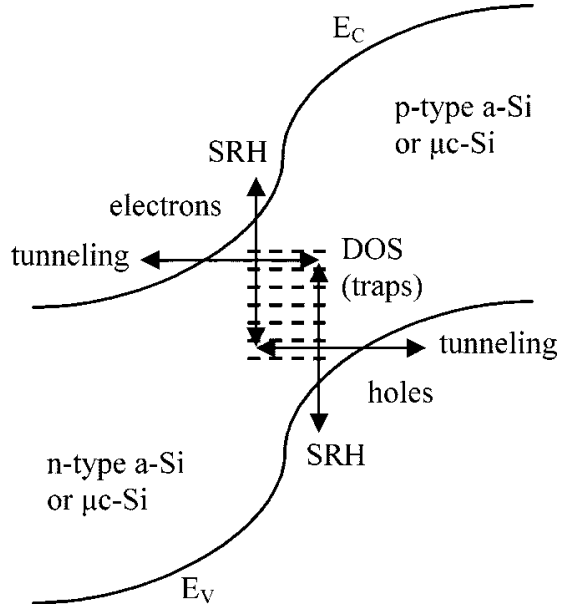

FIG. 1. Schematic representation of the trap-assisted tunneling mechanism (combination of SRH-Shockley-Read-Hall mechanism and tunneling process).

thin barriers previously implemented in D-AMPS involves transitions at constant energy and does not considerably influence the performance of the structures presented in this paper. It differs remarkably from the trap-assisted tunneling of electrons and holes, which is closely linked to the generation and recombination process (energy exchange), as described in the following section.

\section{B. Trap-assisted tunneling models}

To describe tunneling via localized states in the $a-\mathrm{Si}$ mobility gap (Fig. 1), the trap-assisted tunneling theory (TAT) developed by Furlan ${ }^{10}$ was integrated into ASPIN. ${ }^{12}$ TAT is an extension of the SRH model and includes the Poole-Frenkel effect (lowering of the potential barrier caused by the electric field). Similar in essence, however, a completely different numerical model of trap-assisted tunneling based on Hurkx's recombination model ${ }^{9}$ (RM) was integrated into D-AMPS. D-AMPS also accounts for emission enhancement caused by the Poole-Frenkel effect (PFE) ${ }^{7,8}$ not originally included in RM, and allows for two different options: either to work with RM and PFE decoupled but operating simultaneously on the device or to include PFE in electron and hole trap-assisted tunneling models by appropriately modifying Hurkx's algorithms.

Both theories are based on analytical functions that, due to suitable approximations, depend on local variables only (values of variables valid at some other location are expressed with local values; using nonlocal values is known to cause problems with convergence of numerical algorithms). Only one additional independent parameter, the effective tunneling mass $\left(m_{\text {tun }}\right)$, is introduced. Calculated field-dependent factors enlarge the capture cross sections and the emission coefficients of trap states for more intense fields, and the models converge to the SRH equations when the electric field approaches zero.

A straightforward comparison of Hurkx's and Furlan's approach is given in Ref. 10. Let us point out that the former uses Airy functions to estimate the tunneling contribution, while the latter relies on the WKB approximation. Either 



FIG. 2. Estimation of the local electric field and the corresponding (exact or averaged) barrier. In D-AMPS (a), exact local values are taken, whereas in ASPIN (b), the local value of the electric field is taken; however, the barrier is averaged for the WKB approximation.

way, an estimation of the local (exact or averaged) electric field and the corresponding barrier (the conduction band for electrons and the valence band for holes) is required. D-AMPS uses exact local values. On the contrary, in ASPIN only the local value of electric field is taken, while the barrier is averaged for the WKB approximation (Fig. 2). This difference primarily affects the location of the peak and the spread of the tunneling contribution to the generation-recombination process. Recombination rates (forward regime) calculated by D-AMPS are usually a bit higher and limited to a smaller area when compared to ASPIN's output. The described approximations (Airy, WKB) were chosen to reduce calculation times considerably.

\section{ASPIN vs D-AMPS on a $n-i-p \mu c-S i$ TRJ}

In this section we briefly demonstrate that in an actual $n-i-p(\mu c-\mathrm{Si} / \mu c-\mathrm{Si} / \mu c-\mathrm{Si})$ TRJ case ASPIN and D-AMPS give almost identical or at least highly comparable simulation results, in spite of all the differences in the applied models and algorithms. The complete simulation models (TAT + FTM and RM + PFE + FTM) discussed and developed throughout the rest of this paper were applied. A heavily defective intrinsic layer was chosen to amplify the role of traps and the trap-assisted tunneling contribution. Standard material parameters, independently established from other similar simulations and experiments, were used. ${ }^{12}$ Simulated net recombination rates and $J$ - $V$ characteristics of this $n-i-p$ TRJ are compared in Figs. 3 and 4, respectively. This is the only time a $J-V$ plot is shown in a linear scale (later on, we use semilogarithmic plots instead).

The total current in the $\mu c$-Si $n-i-p$ junction is almost completely determined by the trap-assisted tunnel-

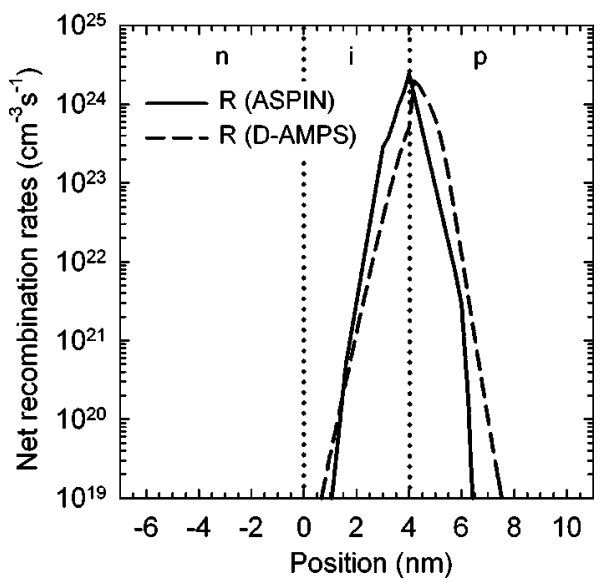

FIG. 3. Net recombination rates at $0.1 \mathrm{~V}$ of an $n-i-p \quad \mu c-\mathrm{Si}$ single TRJ incorporating a defective $4 \mathrm{~nm}$ thick intrinsic layer. Standard material parameters independently established from other similar simulations and experiments were used.

recombination process. The comparison is focused on the forward voltage regime, which corresponds to the working regime of the junction when it is used as a part of a tandem solar cell. Slight discrepancies between the net recombination rates shown in Fig. 3 are mostly due to differences in models implemented in our simulators (Hurkx vs Furlan, different barrier estimations). Predicted $J$ - $V$ characteristics (Fig. 4) reveal a negligible, up to $4 \mathrm{mV}$, deviation in estimated voltage drop over the $n-i-p$ TRJ at the same current level. Such a small difference has no influence on the complete multijunction solar cell simulation result.

TAT and RM +PFE models were proved to be equivalent from the simulation point of view. Simulation results reported in this paper can be obtained by either ASPIN or D-AMPS, and most probably by any other computer program that would incorporate similar models. To make our work completely transparent and consistent, we strictly chose to plot only results obtained with one of the programs (D-AMPS) and tried to keep our discussions as general as possible.

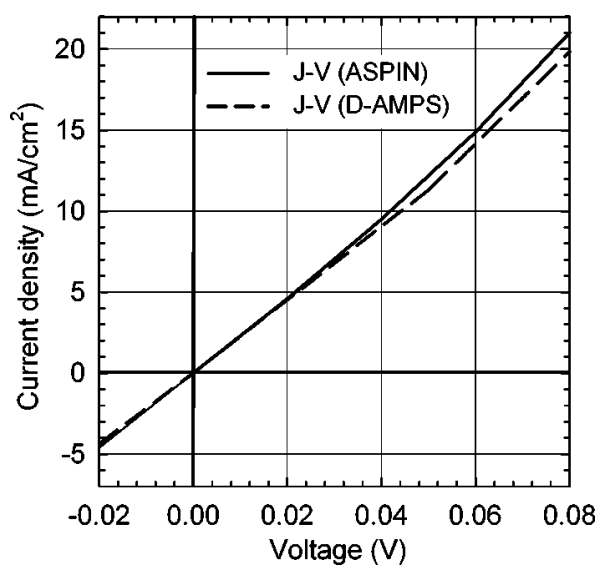

FIG. 4. A comparison of simulated $J-V$ characteristics (on a linear scale) of an $n-i-p \mu c$-Si single TRJ incorporating a defective $4 \mathrm{~nm}$ thick intrinsic layer. Standard material parameters independently established from other similar simulations and experiments were used. 


\section{COMPUTER SIMULATION OF TRJ STRUCTURES}

\section{A. Recombination vs charge trapping}

In the last decade, simulation programs were extensively used for the design and optimization of tandem and triple $a$-Si solar cells. Simulated open circuit voltage $\left(V_{\mathrm{OC}}\right)$ was (initially) always substantially lower than the $V_{\mathrm{OC}}$ of actual devices, and many of the computer-generated $J$ - $V$ curves under illumination showed a "tail" when the applied voltage approached $V_{\mathrm{OC}},{ }^{11,16}$ reflecting the behavior of bad triple junction solar cells.

Under thermodynamic equilibrium, electrons (holes) are the majority carriers on the left (right) side of each $n-p$ TRJ in multiple cells. There is a crossover of free-carrier concentrations at a specific point $x_{c}$ located in the doped layer with the higher activation energy. ${ }^{17}$ The AM1.5 light source gives rise to a large amount of built-up charge and to the excess free carriers inside the TRJ: electrons on the $n$ side and holes on the $p$ side. Free carriers that are photogenerated in the active intrinsic layers and subsequently trapped in TRJ layers introduce light-induced dipoles. Consequently, if the rates of "good" recombination (namely, between electrons and holes photogenerated in the top and bottom $p-i-n$ cell, respectively) are not sufficient, the effective field and the electron barrier of TRJ junctions are lowered. These light-induced dipoles also weaken the electric field inside the $p-i-n$ intrinsic layers, causing a lot of "bad" recombination (between electrons and holes photogenerated in the same $p-i-n$ cell), which reduces the total current.

The classical SRH recombination model is not able to provide enough good recombination in the TRJ structures to favor recombination over trapping (i.e., electrons and holes pile up and form light-induced dipoles), which leads to a substantial voltage drop in TRJ junctions. Consequently, the current levels at voltages near $V_{M}$ (maximum power point) are significantly lowered. The increase in the density of states in the TRJs would strengthen these light-induced dipole effects even more. Within the SRH formalism, the reduction of light-induced dipoles could be accomplished by adopting very large capture cross sections, more than ten orders larger than normally assumed. ${ }^{16}$

Hou found an alternative solution in decreasing the simulated $a$-Si mobility band gap around $x_{c}$ to values as low as $1.1 \mathrm{eV} .^{16}$ To further ease the recombination between electrons and holes in this region, they also graded the conduction band of the $n$ layer and the valence band of the $p$ layer. Although this approach accounts for the effects of the space charge on the band bending in TRJ, it is difficult to properly model the distribution of localized states and their influence on device behavior. At the same time, there is no experimental evidence supporting a band gap lowering inside a TRJ. Band gap grading is not needed in TRJs where all layers are of $\mu c-\mathrm{Si}$ if its mobility band gap is assumed to be equal to the band gap of monocrystalline silicon $(c-\mathrm{Si}){ }^{17}$ Applying Hurkx's RM and the PFE model simultaneously, the experimental $V_{\mathrm{OC}}$ of $a$-Si $/ a$-Si tandem solar cells with $\mu c$-Si TRJ could be reproduced with D-AMPS for $\mu c$-Si mobility gaps up to $1.4 \mathrm{eV} .^{17,18}$ Furthermore, Willemen et al..$^{5}$ successfully fitted $V_{\mathrm{OC}}$ of $a$-Si-based tandem solar cells using standard val- ues (1.7-2.0 eV) for the $a$-Si mobility gap of the TRJ layers, which will be discussed in the following sections.

When recombination plus trap-assisted tunneling (which is also a recombination process) versus charge trapping is addressed, different ways of modeling of material properties can influence simulated device characteristics considerably more in comparison with devices where the standard SRH model is accurate enough. As will be described later, we used two contrasting descriptions of $\mu c-\mathrm{Si}$ DOS and activation energies of $a$-SiC to highlight reasons for different device performances, which should also be considered during device design and optimization. Wide tails and low dangling bond (DB) density, for example, can lead to higher recombination rates than the opposite case of steep tails and high DB density, because charge carriers that are already far from their corresponding bands are more likely to recombine than to be reemitted (e.g., an electron trapped in a donorlike tail state near the valence band edge is more likely to be recombined with a hole from the valence band then to be reemitted to the conduction band); the trapped charge balance is also changed. Of course, there are a number of parameters that are important in this evaluation, such as individual trap capture and emission cross sections, charged vs neutral cross section ratio, etc.

\section{B. Mechanisms controlling the total current in TRJs}

In a power generating tandem solar cell $(p-i-n-p-i-n$, $p-i-n-i-p-i-n)$, the TRJ is an $n-p$ or $n-i-p$ forward biased structure. For a well designed TRJ the voltage drop over the junction $\left(V_{\mathrm{TRJ}}\right)$ has to be very small. Our simulations show that in $a-\mathrm{Si} / a-\mathrm{Si}$ tandem solar cells with efficiencies over $10 \%, V_{\text {TRJ }}$ is not higher than $0.05 \mathrm{~V}$. If $V_{\text {TRJ }}$ of $0.3 \mathrm{~V}$ is assumed (bad TRJ), for instance, not more than $8 \%$ efficiency can be achieved. Electrons (holes) injected into the $n$ layer ( $p$ layer) move by diffusion against the field. As they cannot surmount the high built-in potential present in TRJ, they have to recombine (the high built-in potential must exist and is essential for obtaining high $V_{\mathrm{OC}}$, which is the sum of voltages over both $p-i-n$ subcells). The recombination rate profile shows a sharp peak in TRJ around the position where the Fermi level crosses the midgap.

Recombination rates limit the total current in lowforward biased $n-p$ or $n-i-p$ junctions when the recombination process is described by the SRH formalism. ${ }^{19}$ If standard electrical parameters are assumed for TRJ structures regularly used in $a$-Si multiple junction cells, our simulation programs predict current levels in the range from 2 $\times 10^{-7} \mathrm{~mA} / \mathrm{cm}^{2}$ to $5 \times 10^{-6} \mathrm{~mA} / \mathrm{cm}^{2}$ at a forward voltage of $0.05 \mathrm{~V}$. A typical current level in $a$-Si tandem solar cells is about $8 \mathrm{~mA} / \mathrm{cm}^{2}$, and Hegedus et al. ${ }^{4}$ reported experimental $J-V$ curves of several TRJs produced as single junction devices with currents ranging from $1 \mathrm{~mA} / \mathrm{cm}^{2}$ to $3 \mathrm{~mA} / \mathrm{cm}^{2}$ at $0.05 \mathrm{~V}$. This means that calculated current levels have to be increased by seven orders of magnitude (by applying proper physical models). As pointed out before, we would need to enlarge the capture cross sections within the SRH formalism to unreasonably large values to match the experimentally observed currents. Moreover, computer generated $J$ - $V$ curves of 
$n-p$ and $n-i-p$ structures are rectifying, while the measured $J$ - $V$ curves published by Hegedus et al. show similar current levels for reverse and forward voltages. These facts clearly indicate that we have to include an extra transport mechanism in order to enhance recombination.

The physics controlling the charge transport in isolated (produced as single junction devices) TRJ structures and the physics of TRJs being part of multiple junction solar cells are similar, but some differences can be pointed out. In both cases, there is injection of electrons and holes from the contacts into the $n$-layer, and $p$-layer, respectively, diffusion to higher potential energies, and finally recombination. In single TRJ junctions the dark current supply can be limited by doped layer conductivities or by bad contacts. In multiple junctions the current supply, i.e., the injected current, could additionally be limited by recombination losses taking place in the intrinsic layers of the $p-i-n$ subcells. In isolated TRJ there is also a charge build-up caused by current injection. We could identify this charge trapped in doped layers by the name "injection-induced dipole." However, this dipole does not seriously affect the $n-p$ or the $n-i-p$ effective built-in potential barrier, because the barrier is controlled by the activation energies of doped layers or by contacts when doped layers are not heavily doped (and, of course, by the applied voltage). Under normal working conditions the current levels in multiple junction solar cells under illumination are higher than in a single TRJ for the same applied voltage.

To simplify the discussion, isolated TRJ structures will be studied first. In this paper, five different TRJs combining $a-\mathrm{Si}, a-\mathrm{SiC}$, and $\mu c$-Si layers are examined. Next, the following structure is discussed in detail: $n-p^{+}-p(a-\mathrm{Si} / a-\mathrm{Si} / a$ -SiC; $25 \mathrm{~nm} / 5 \mathrm{~nm} / 25 \mathrm{~nm}$ ). Finally, dark $J-V$ curves of all proposed TRJ structures are compared and discussed.

\section{Enhancing recombination in TRJs}

As the SRH formalism in our computer codes predicts very low current levels for the working regime voltages, we had to include additional mechanisms to enhance the recombination. We explored several alternatives (already discussed): RM, ${ }^{9}$ with and without PFE, ${ }^{7,8} \mathrm{TAT},{ }^{10}$ as well as a model predicting field-dependent capture and emission rates (FCE) discussed below.

RM and TAT define analytical derivations that introduce field-dependent factors enlarging the capture cross sections and the emission coefficients of trap states. Strong electric fields $\left(F>10^{4} \mathrm{~V} / \mathrm{cm}\right)$ increase the transparency of potential barriers opposing the tunneling of free carriers from extended states toward gap states. Expressions converge to the SRH equations when the electric field approaches zero. The formalism of RM and TAT is sometimes referred to as recombination tunneling, since tunneling is taken into account by enhancing the recombination.

In the PFE model the effective cross sections of charge defects become field dependent. They are enhanced by the factor $E_{H}=\exp \left(\Delta E_{I} / k T\right)$, where $\Delta E_{I}$ is given by $\Delta E_{I}$ $=q(q F / \pi \varepsilon)^{1 / 2}$ resembling the well-known equation of image lowering. The symbol $F$ stands for the electric field strength in the device at the grid point under consideration (local value). Some authors argue that not only cross sections of

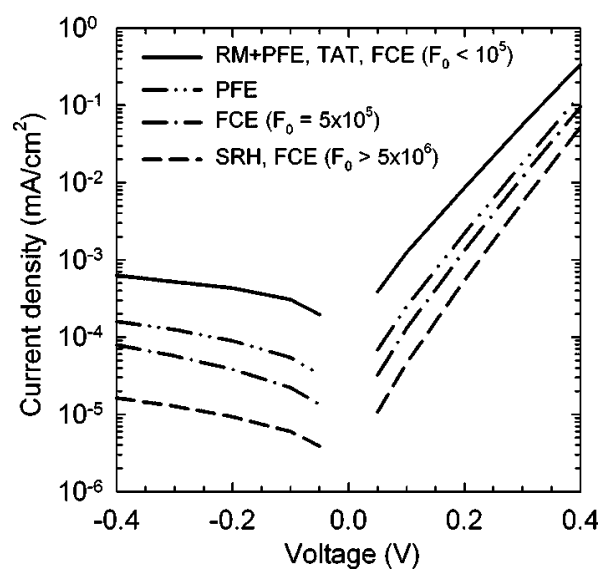

FIG. 5. Dark $J-V$ characteristics of an $n-p^{+}-p(a-\mathrm{Si} / a-\mathrm{Si} / a-\mathrm{SiC}) \mathrm{TRJ}$ obtained using different models that enhance capture cross sections and emission coefficients: SRH, FCE $\left(F_{0}\right.$ in V/cm $)$, PFE, RM+PFE $\left(m_{\text {tun }}=0.01 m_{\mathrm{e}}\right.$, $\left.\mu_{n}=20 \mathrm{~cm}^{2} / \mathrm{Vs}, \mu_{p}=2 \mathrm{~cm}^{2} / \mathrm{Vs}\right)$. TAT model is approximately equivalent to balanced RM+PFE.

charged traps, but also neutral cross sections are enlarged by the same factor $E_{H} \cdot{ }^{10}$ Both possibilities were considered in our analysis.

The FCE model is based on conclusions similar to the predictions of $\mathrm{Gu}$ and Schiff. ${ }^{20}$ Simulation results, as well as the RM and TAT formalisms, lead to the conclusion that the attempt-to-escape frequency is field dependent in the form of $\nu=\nu_{0} \exp \left(F / F_{0}\right)$. However, in spite of the reasoning of Gu and Schiff and following the idea of RM and TAT, we implemented field-dependent cross sections as $\sigma(F)=\sigma_{0} \exp \left(F / F_{0}\right)$, where $F_{0}$ is an input parameter $(\mathrm{V} / \mathrm{cm})$. Instead, the effective density of state at the mobility edge $\left(N_{C}, N_{V}\right)$ was kept independent of electric field strength.

Dark $J-V$ curves obtained for the different models just described are plotted in Fig. 5.

Pushing simulation parameters to their limits (either by lowering effective tunneling masses, making PFE affect charged and neutral capture and emission cross sections, or lowering values of $F_{0}$ in FCE) increases the forward and reverse currents only to a certain extent. The $J$ - $V$ curves remain rectifying, which contradicts measurement results. For instance, with FCE we were able to enhance the total current in an $n-p^{+}-p \quad(a-\mathrm{Si} / a-\mathrm{Si} / a-\mathrm{SiC})$ TRJ up to $4 \times 10^{-4} \mathrm{~mA} / \mathrm{cm}^{2}$ at a forward voltage of $0.05 \mathrm{~V}$. We observed that the current cannot be increased any further by decreasing $F_{0}$ below $5 \times 10^{4} \mathrm{~V} / \mathrm{cm}$.

In order to reach the measured current levels, we need to question the charge transport mechanisms. Whichever model is chosen (TAT, RM, PFE, FCE), the effective cross sections become strongly field dependent, and they are significantly enhanced with respect to the values used in the original SRH formalism. In all of these models, we are really working on a single grid point, and there is no real connection in the sense of lateral charge movement between the regions where the recombination is maximal and the regions where free-carrier concentrations are high (the origins of tunneling). Although the described models enhance recombination for increasing electric fields, electrons and holes still have to move to the physical position where recombination takes place. 


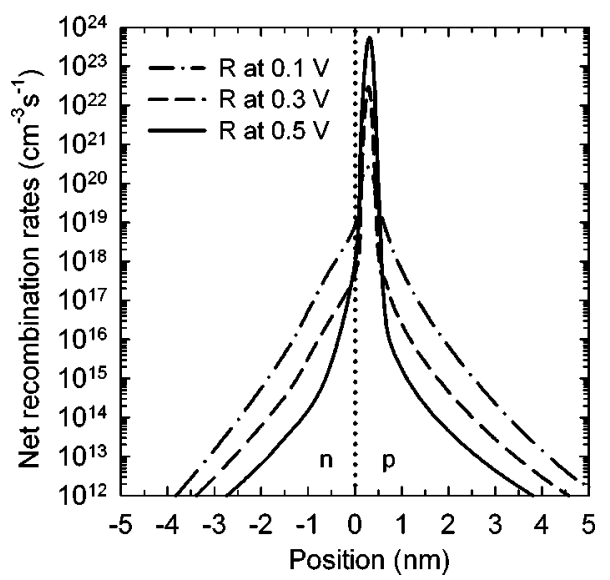

FIG. 6. Recombination rates $(R)$ in an $n-p(a-\mathrm{Si} / a-\mathrm{SiC}, 25 \mathrm{~nm} / 25 \mathrm{~nm}) \mathrm{TRJ}$ at different forward biases. Vicinity of the metallurgic junction is shown; $R<10^{12} \mathrm{~cm}^{-3} \mathrm{~s}^{-1}$ elsewhere.

An increase of free-carrier mobilities helps diffusion of majority free carriers against the electric field (via Einstein's relation) to the region where they actually recombine. Willemen has already suggested this possibility in his Ph.D thesis. ${ }^{11}$ We checked that the front and the back contacts and the $n$-layer and $p$-layer conductivities are not limiting the total current. It is interesting that the same conclusions were drawn for D-AMPS using Hurkx's RM and for ASPIN using the TAT model based on Furlan's theory. Models compensating for the lack of sufficient charge flow by enhancing the extended state mobility were successfully tested.

\section{Impact of using high mobilities on charge transport in TRJs}

In order to locate areas where the total current is limited by diffusion, we increased mobilities for both electrons and holes by the same factor in different regions of the simplest TRJ structure studied by Hegedus, ${ }^{4}$ the $n-p(a-\mathrm{Si} / a-\mathrm{SiC})$ junction. We found that the total current did not change when we increased the mobilities in the $n$-type $(a$-Si) and $p$-type $(a-\mathrm{SiC})$ layers near the front and back contacts only. The total current started to rise when we increased the mobilities in the region where the recombination rate reaches $10^{-3}$ of its peak value. The recombination peak is located close to the $n-p$ interface and coincides with the crossover between the electron and hole free-carrier concentrations. Only when both mobilities are increased by the same factor $\left(\mathrm{f}_{\mu}\right)$ over entire structure do we see a parallel displacement in the current level $(J)$ by this factor $\left(\mathrm{f}_{\mu}\right)$ in the voltage range of interest $(-0.3 \mathrm{~V},+0.3 \mathrm{~V})$. Corresponding recombination rates at different applied forward voltages under dark conditions are shown in Fig. 6. Increasing both mobilities with higher and higher values of $f_{\mu}$, we observe a parallel displacement of the entire $J-V$ curve as long as $\mathrm{f}_{\mu}$ does not exceed $10^{5}$. Surpassing this value, contacts or doped layer conductivities start to limit the total current. If we continue with the simulation experiment and set the barriers at both contacts to only $0.01 \mathrm{eV}$ and if keep increasing the doping levels up to $10^{21} \mathrm{~cm}^{-3}$ (both semiconductors become degenerated; the Fermi level is around $0.1 \mathrm{eV}$ inside bands), we can observe that $J-V$ curves scale up perfectly for higher mobilities - up

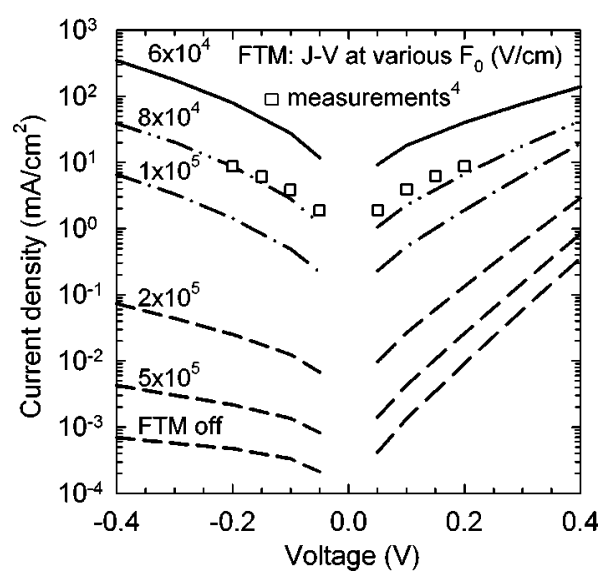

FIG. 7. Dark $J-V$ characteristics of an $n-p^{+}-p(a-\mathrm{Si} / a-\mathrm{Si} / a-\mathrm{SiC})$ TRJ calculated assuming that both mobilities follow the expression $\mu=\mu_{0} \exp \left[F(V, x) / F_{0}\right], \mu=f\left[\mu_{0}(x), F(V, x), T, F_{0}\right]$, i.e., the FTM model.

to a factor of $10^{9}$. In order to reach experimentally observed current levels, mobilities have to be scaled up by at least a factor of $10^{4}$ all over the device. The original extended state electron and hole mobilities were assumed to be 20 and $2 \mathrm{~cm}^{2} / \mathrm{V} \mathrm{s}$, respectively.

There is experimental evidence suggesting that conductivity and drift mobility in $a$-Si increase exponentially with the electric field $F^{21,22}$ In spite of the fact that transport in TRJ is by diffusion (mobilities and diffusion coefficients are connected through Einstein's relation) and that experimental results published in the literature are related to the transport of electrons by drift, Willemen assumed ${ }^{5,11}$ that both extended state electron and hole mobilities are exponentially dependent on the electric field, according to the expression $\mu(F)=\mu_{0} \exp \left(F / F_{0}\right)$, where $F_{0}$ is a parameter determining the magnitude of the dependence on electric field strength. In Willemen's original model, mobilities were recalculated only once, with the values obtained from the simulation results under thermal equilibrium conditions, so we should rewrite the expression above as $\mu(F)=\mu_{0} \exp \left(F_{\mathrm{TE}} / F_{0}\right)$. The temperature dependence of the model was never addressed. With such an approach, the quality of a TRJ can easily be overestimated. In badly designed TRJs, the electric field can vary considerably with the working conditions (voltage, temperature, etc.) and these dependencies should also be modeled.

To make the simulations coherent, we implemented a similar model (FTM - field and temperature dependent mobility) but recalculated mobilities in each iteration of the simulation, making mobility field and position dependent: $\mu=\mu_{0} \exp \left[F(V, x) / F_{0}\right]$, more precisely $\mu$ $=f\left[\mu_{0}(x), F(V, x), T, F_{0}\right]$. Expressions involving mobilities were integrated into the preexisting equation set so that the entire system of semiconductor equations could be selfconsistently solved by the iterative algorithm. The feedback effect of the FTM model on other device properties was therefore not neglected.

Returning now to the more complex structure $n-p^{+}-p$ $(a-\mathrm{Si} / a-\mathrm{Si} / a-\mathrm{SiC})$, Fig. 7 shows the dark $J-V$ curves of the $n-p^{+}-p$ structure obtained for different values of $F_{0}$. In order to reach the currents measured by Hegedus et al. $\left(\sim 3 \mathrm{~mA} / \mathrm{cm}^{2}\right.$ at $\left.\mathrm{V}=0.05 \mathrm{~V}\right)$, the value of the parameter $F_{0}$ 
TABLE I. Simulation parameters of the five TRJ structures. Two different sets of parameters $\left(\mathrm{ST}_{\mu c}\right.$ and $\left.\mathrm{WT}_{\mu c}\right)$ have been used for $\mu c$-Si.

\begin{tabular}{lcccccc}
\hline \hline & $n(a-\mathrm{Si})$ & $p^{+}(a-\mathrm{Si})$ & $p(a-\mathrm{SiC})$ & $n(\mu c-\mathrm{Si})$ & $i(\mu c-\mathrm{Si})$ & $p(\mu c-\mathrm{Si})$ \\
\hline Thickness $(\mathrm{nm})$ & 25 & $10^{\mathrm{a}}$ & 25 & 20 & 5 & 20 \\
Activation energy $(\mathrm{eV})$ & 0.27 & $0.2^{\mathrm{a}}$ & $0.47^{\mathrm{b}}$ & 0.026 & 0.73 & 0.059 \\
Mobility gap (eV) & 1.76 & 1.72 & 1.98 & 1.54 & 1.54 & 1.54 \\
\hline \multirow{2}{*}{ DB density $\left(\mathrm{cm}^{-3}\right)$} & $5 \times 10^{18}$ & $6 \times 10^{18}$ & $5 \times 10^{18}$ & $5 \times 10^{18}$ & $1.5 \times 10^{18}$ & $5 \times 10^{18}$ \\
& & & & $5 \times 10^{16}$ & $5 \times 10^{15}$ & $5 \times 10^{16}$ \\
Tail slope $E_{a} / E_{d}(\mathrm{meV})$ & $30 / 50$ & $40 / 60$ & $45 / 80$ & $10 / 10$ & $10 / 10$ & $10 / 10$ \\
& & & & $20 / 30$ & $20 / 30$ & $20 / 30$ \\
$G_{d o}, G_{a o}\left(\mathrm{~cm}^{-3} \mathrm{eV}^{-1}\right)$ & $10^{21}$ & $10^{21}$ & $10^{21}$ & $2 \times 10^{20}$ & $2 \times 10^{20}$ & $2 \times 10^{20}$ \\
Mobilities $\left(\mathrm{cm}^{2} / \mathrm{V} \mathrm{s}\right)$ & $20 / 2$ & $20 / 2$ & $20 / 2$ & $40 / 4$ & $10^{21}$ & $10^{21}$ \\
\hline \hline
\end{tabular}

${ }^{\mathrm{a}}$ Results of simulations following more faithfully the parameter values and thicknesses as reported by Hegedus et al. (Ref. 4) were already presented in Ref. 12.

${ }^{\mathrm{b}}$ Devices with a more optimistic activation energy of $0.33 \mathrm{eV}$ will be discussed as well.

had to be kept below $8 \times 10^{4} \mathrm{~V} / \mathrm{cm}$. Interestingly, we see in the same figure that the predicted dark $J$ - $V$ curve is almost Ohmic (at $\mathrm{F}_{0}=8 \times 10^{4} \mathrm{~V} / \mathrm{cm}$ ), as was experimentally observed.

\section{Comparison of different TRJ structures}

The following five devices will be examined in this section: (1) $n-p^{+}-p(a-\mathrm{Si} / a-\mathrm{Si} / a-\mathrm{SiC}),(2) n-p(a-\mathrm{Si} / a-\mathrm{SiC}),(3)$ $n-p^{+}-p(\mu c-\mathrm{Si} / a-\mathrm{Si} / a-\mathrm{SiC}),(4) n-i-p(\mu c-\mathrm{Si} / \mu c-\mathrm{Si} / \mu c-\mathrm{Si})$ and (5) $n-p(\mu c-\mathrm{Si} / \mu c-\mathrm{Si})$. Thicknesses, activation energies, mobility gaps, baseline mobilities $\left(\mu_{0}\right)$, and DOS of different materials utilized in our simulations are listed in Table I. As there is uncertainty in the literature about the electrical parameters of $\mu c-\mathrm{Si}$, we decided to model this material with two different sets of inputs: the $\mathrm{ST}_{\mu c}$ set corresponds to a high dangling bond (DB) density and steep tails and the $\mathrm{WT}_{\mu c}$ set to a low DB density and wide tails semiconductor.

Band offsets in heterojunctions were split half and half between the conduction and valence bands. The value of the tunneling effective mass was defined as $0.01 m_{e}$, where $m_{e}$ is the free electron mass.

Flat band conditions at both contacts were assumed; therefore, the overall built-in potential is obtained as the difference in energy between the bulk Fermi levels of the semiconductor materials located at the front and at the back contact. Overall built-in potentials $\left(V_{\mathrm{BI}}\right)$ and interface built-in potentials of the five structures are shown in Table II. Two of these structures contain one interface and three of them contain two interfaces. In the second case, Table II also indicates the built-in potential present at the left and at the right interfaces. These figures correspond to the energy difference between Fermi levels of individual layers before each junction is made. Positive values are used when the energy of the Fermi level corresponding to the semiconductor located at the left-hand side of the interface is higher than the energy of the Fermi level of the semiconductor located at the righthand side of the interface.

The first two structures have the same total built-in potential. Nevertheless, the electric field profiles reveal important differences (Fig. 8). In the first structure the $n-p^{+}$and the $p^{+}-p$ interfaces give rise to electric fields of opposite directions. The electric field is reinforced at the $n-p^{+}$interface, and it is weakened at the $p^{+}-p$ interface (see Table II). However, differences between the electric field profiles of structures (1) and (2) are minor in comparison to the electric field profiles of the other three structures where built-in potentials are higher. The electric field at the left interface in structure (3) is considerably higher than its counterpart in structure (4) (Fig. 8). Field profiles are quite important in our discussion because the mobility is assumed to be exponentially dependent on the electric field strength. Figure 9 shows the dark $J-V$ characteristics at room temperature for $F_{0}=10^{5} \mathrm{~V} / \mathrm{cm}$. Mobilities were recalculated according to the FTM model.

The following mechanisms are acting in series when TRJs are forward biased: electron injection through the front contact, diffusion of electrons against the field, tunneling of electrons from extended to gap states, recombination of electrons and holes in gap states, tunneling of holes from extended to gap states, diffusion of holes against the field, and

TABLE II. Built-in potentials of simulated TRJs and potential barriers present at each interface of TRJ structures having more than one interface.

\begin{tabular}{|c|c|c|c|c|c|}
\hline & 1 & 2 & 3 & 4 & 5 \\
\hline Structure & $n-p^{+}-p$ & $n-p$ & $n-p^{+}-p$ & $n-i-p$ & $n-p$ \\
\hline Materials & $a-\mathrm{Si} / a-\mathrm{Si} / a-\mathrm{SiC}$ & $a-\mathrm{Si} / a-\mathrm{SiC}$ & $\mu c-\mathrm{Si} / a-\mathrm{Si} / a-\mathrm{SiC}$ & $\mu c-\mathrm{Si}$ & $\mu c-\mathrm{Si}$ \\
\hline Overall $V_{\mathrm{BI}}(\mathrm{eV})$ & 1.1 & 1.1 & 1.244 & 1.455 & 1.455 \\
\hline Left $V_{\mathrm{BI}}(\mathrm{eV})$ & 1.29 & & 1.424 & 0.77 & \\
\hline Right $V_{\mathrm{BI}}(\mathrm{eV})$ & -0.18 & & -0.18 & 0.685 & \\
\hline
\end{tabular}




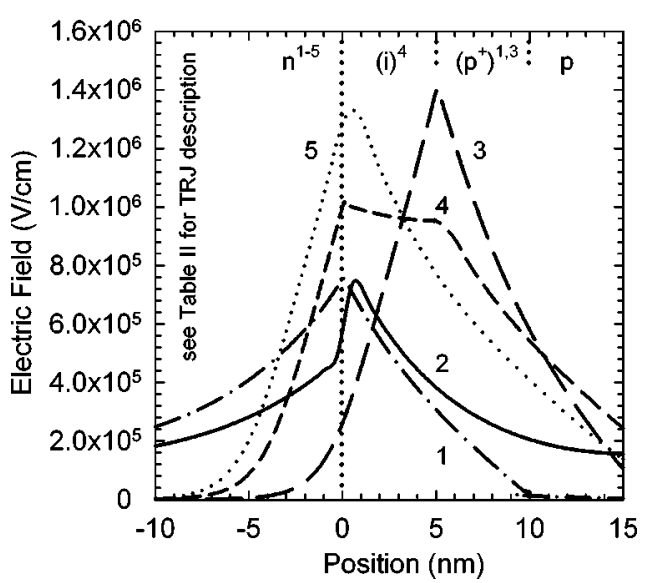

FIG. 8. Electric field profiles of different TRJs at a forward voltage of $0.1 \mathrm{~V}$. Structures are aligned to the $n$-layer metallurgic junction position at $0 \mathrm{~nm}$ (see Table II; $\mathrm{ST}_{\mu c}$ DOS was assumed for $\mu c$-Si layers).

hole injection through the back contact. The total current is defined by the limiting mechanism. Interestingly, in all of these five different devices-for the parameters adopted and assuming $\mathrm{ST}_{\mu c}$ layers-we find that the same mechanism is limiting the total current: diffusion against the electric field, i.e., free-carrier concentrations and low mobilities are the simulated current bottlenecks. Although hole diffusion is more limiting than electron diffusion, both carriers play their role in defining the total current. In addition, tunneling and recombination (recombination in $\mu c$-Si layers is dominated by midgap states due to its low mobility gap) also play a minor role in limiting the total current in devices made entirely of $\mu c-\mathrm{Si}$. On the contrary, the total current in the $n$ $-p^{+}-p(\mu c-\mathrm{Si} / a-\mathrm{Si} / a-\mathrm{SiC}) \mathrm{TRJ}$ is limited purely by diffusion, regardless of DOS distribution (either $\mathrm{ST}_{\mu c}$ or $\mathrm{WT}_{\mu c}$ ) in the $\mu c$-Si layer.

Observed simulation trends agree well with the experimental results published by Hegedus. ${ }^{4} J-V$ curves of different TRJs shown in Fig. 9 were calculated for the same value of the parameter $F_{0}\left(10^{5} \mathrm{~V} / \mathrm{cm}\right)$. For lower or higher values of $F_{0}$, the experimental trend is still preserved. The quantitative reproduction of Hegedus' results requires, on the other hand, certain dispersion in $F_{0}$ for the three TRJs (depending



FIG. 9. Dark $J$ - $V$ characteristics predicted for the five TRJs under study (see Table II). For easier comparison FTM parameter $F_{0}$ was always set to $10^{5} \mathrm{~V} / \mathrm{cm}$. on all other material parameters used in the simulation): 8 $\times 10^{4} \mathrm{~V} / \mathrm{cm}$ in the $n-p^{+}-p \quad(a-\mathrm{Si} / a-\mathrm{Si} / a-\mathrm{SiC}), \quad 5.5$ $\times 10^{4} \mathrm{~V} / \mathrm{cm}$ in the $n-p(a-\mathrm{Si} / a-\mathrm{SiC})$, and $1 \times 10^{5} \mathrm{~V} / \mathrm{cm}$ in the $n-p^{+}-p(\mu c-\mathrm{Si} / a-\mathrm{Si} / a-\mathrm{SiC})$ cases.

We have assumed in our simulations that the $(a-\mathrm{SiC})$ $p$-layer has an activation energy $\left(E_{a}\right)$ of $0.47 \mathrm{eV}$. This high activation energy can lead to a device where the depletion region would spread beyond the given location of the corresponding metal contact if it could be possible. The performance of such a TRJ can be improved by lowering the activation energy of the $a$-SiC layer. We observed a significant increase (one order, in the case of $E_{a}=0.33 \mathrm{eV}$ ) in the total current of the $n-p(a-\mathrm{Si} / a-\mathrm{SiC})$ and $n-p^{+}-p(\mu c-\mathrm{Si} / a-\mathrm{Si} / a-$ $\mathrm{SiC})$ TRJ structures. Only a minor increase in the current is obtained in the $n-p^{+}-p(a-\mathrm{Si} / a-\mathrm{Si} / a-\mathrm{SiC})$ TRJ because the recombination peak is located well inside the $(a-\mathrm{Si})$ $n$-layer, the $n-p$ junction is formed with the $(a-\mathrm{Si}) p^{+}$layer, and therefore the $(a-\mathrm{SiC}) p$ layer contributes mostly to the serial resistance of the device. Hegedus' measurements can be matched with a simulation based on lower activation energy $(0.33 \mathrm{eV})$ without major changes in the parameter $F_{0}$ for all three TRJ devices: $8 \times 10^{4} \mathrm{~V} / \mathrm{cm}$ in $n-p^{+}-p$ $(a-\mathrm{Si} / a-\mathrm{Si} / a-\mathrm{SiC}), 1 \times 10^{5} \mathrm{~V} / \mathrm{cm}$ in $n-p(a-\mathrm{Si} / a-\mathrm{SiC})$, and $1 \times 10^{5} \mathrm{~V} / \mathrm{cm}$ in $n-p^{+}-p(\mu c-\mathrm{Si} / a-\mathrm{Si} / a-\mathrm{SiC})$.

In the $n-i-p(\mu c-\mathrm{Si} / \mu c-\mathrm{Si} / \mu c-\mathrm{Si})$ and $n-p(\mu c-\mathrm{Si} / \mu c-$ $\mathrm{Si}) \mathrm{TRJ}$ structures (not studied by Hegedus), simulations predict significantly higher total currents due to a much larger supply of free holes provided by the $(\mu c-\mathrm{Si}) p$ layer compared to the $(a-\mathrm{Si}) p$ layer. $\mathrm{WT}_{\mu c}$ layer $n-i-p$ and $n-p$ devices show even higher currents due to stronger electric fields formed in these junctions. Thus, field-dependent mobilities and consequently diffusion against the electric field increase significantly. A decrease in DB densities improves the electric field (and mobilities that are field dependent) at the junction by shielding (with trapped charge) the field originating from the charge of active dopants with less intensity. The removal of the intrinsic layer helps in shaping the electric field that otherwise spreads over the whole intrinsic layer. This explains the results shown in Fig. 9, where higher currents are calculated for devices with $\mathrm{WT}_{\mu c}$ layers (less DB) than devices with $\mathrm{ST}_{\mu c}$ layers (more $\mathrm{DB}$ ). This result also confirms the fact that simulated total currents are (normally) not limited by recombination when TAT, RM, PFE, or FCE are applied. However, the value of $F_{0}$ could as well be underestimated in the case of the $\mathrm{WT}_{\mu c}$ simulation parameter set. We found no experimental evidence reported in the literature that would confirm or overthrow either of the conclusions.

Simulations presented in this paper indicate that structures entirely made of $\mu c$-Si are the least current limiting of the five analyzed structures when functioning as TRJs and are therefore most promising for implementation in multijunction solar cells.

\section{COMPARISON OF DIFFERENT TRJ STRUCTURES IN a-Si/a-Si TANDEM CELLS}

In this section we study the dependence of the performance of an $a-\mathrm{Si} / a-\mathrm{Si}$ tandem solar cell with respect to the 
TABLE III. Efficiency (\%) of the $a$-Si/a-Si tandem solar cell for different TRJ structures and for different values of $F_{0}$. In the last line, only the RM + PFE contribution is considered, i.e., the physics of field-dependent mobilities described by the FTM model is removed. Both WT $\mathrm{Wc}_{c}$ and $\mathrm{ST}_{\mu \mathrm{c}} \mathrm{DOS}_{\mathrm{Sarameter}}$ sets for $\mu c$-Si layers are accounted for. The activation energy in the $(a-\mathrm{SiC}) p$ layer is assumed to be either $0.33 \mathrm{eV}\left(\mathrm{LE}_{\mathrm{a}}\right)$ or $0.47 \mathrm{eV}\left(\mathrm{HE}_{\mathrm{a}}\right)$.

Tandem solar cell efficiency (\%) with respect to the TRJ used

\begin{tabular}{lccccc}
\cline { 2 - 6 }$F_{0}(\mathrm{~V} / \mathrm{cm})$ & $n-p\left(\mathrm{ST}_{\mu c}\right)^{\mathrm{a}}$ & $n-p\left(\mathrm{WT}_{\mu c}\right)^{\mathrm{a}}$ & $n-i-p\left(\mathrm{ST}_{\mu c}\right)^{\mathrm{a}}$ & $n-i-p\left(\mathrm{WT}_{\mu c}\right)^{\mathrm{a}}$ & $n-p^{+}-p\left(\mathrm{LE}_{\mathrm{a}}\right)^{\mathrm{b}}$ \\
\hline $8 \times 10^{4}$ & 11.46 & 11.62 & 11.45 & 11.57 & 11.57 \\
$1 \times 10^{5}$ & 11.45 & 11.62 & 11.43 & 9.70 & 11.40 \\
$5 \times 10^{5}$ & 9.52 & 9.87 & 9.25 & 9.27 & 8.59 \\
$1 \times 10^{6}$ & 9.20 & 9.27 & 8.96 & 8.67 & 8.30 \\
FTM off & 8.87 & 8.74 & & 8.04
\end{tabular}

\begin{tabular}{lccccc}
$F_{0}(\mathrm{~V} / \mathrm{cm})$ & $n-p^{+}-p\left(\mathrm{HE}_{\mathrm{a}}\right)^{\mathrm{b}}$ & $n-p^{+}-p\left(\mathrm{HE}_{\mathrm{a}}\right)^{\mathrm{c}}$ & $n-p^{+}-p\left(\mathrm{LE}_{\mathrm{a}}\right)^{\mathrm{c}}$ & $n-p\left(\mathrm{HE}_{\mathrm{a}}\right)^{\mathrm{c}}$ & 5.58 \\
\hline $6 \times 10^{4}$ & 9.98 & 8.05 & 9.40 & 5.20 & 8.33 \\
$8 \times 10^{4}$ & 9.75 & 7.21 & 8.53 & 5.03 & 7.38 \\
$1 \times 10^{5}$ & 9.17 & 6.93 & 8.10 & 4.61 & 6.96 \\
$5 \times 10^{5}$ & 7.31 & 6.03 & 6.86 & 4.57 & 6.03 \\
$1 \times 10^{6}$ & 7.11 & 5.91 & 6.74 & 4.52 & 5.88 \\
FTM off & 6.95 & 5.80 & 6.63 & & 5.86 \\
\hline
\end{tabular}

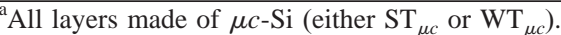

${ }^{\mathrm{b}} n$ made of $\mu c-\mathrm{Si}, p^{+}$of $a-\mathrm{Si}, p$ of $a-\mathrm{SiC}$ (either $\mathrm{HE}_{\mathrm{a}}$ or $\mathrm{LE}_{\mathrm{a}}$ ).

${ }^{c} n$ and $p^{+}$made of $a-\mathrm{Si}, p$ of $a-\mathrm{SiC}$ (either $\mathrm{HE}_{\mathrm{a}}$ or $\mathrm{LE}_{\mathrm{a}}$ ).

TRJ structure selected to connect two $p-i-n$ subcells. We focus our discussion on the $a-\mathrm{Si} / a$-Si device having mobility gaps of $1.88 \mathrm{eV}$ and $1.78 \mathrm{eV}$ that has been produced, characterized, and previously modeled at Utrecht University. ${ }^{17,18}$ The experimental efficiency of these cells was slightly above $9 \%$, independent of the TRJ structure used: $n-i-p(\mu c-\mathrm{Si} /$ $\mu c-\mathrm{Si} / \mu c-\mathrm{Si})$ or $n$-ox- $p(\mu c-\mathrm{Si} /$ oxide/ $\mu c-\mathrm{Si})$; the simulated $\mu c$-Si mobility gap was assumed to be $1.2 \mathrm{eV}$, and for the effective tunneling mass, the value of the extended state effective mass of $c$-Si was used. ${ }^{17}$ By lowering the effective tunneling mass to $0.01 m_{e}$, where $m_{e}$ is the free electron mass, tandem solar cell efficiencies up to $10 \%$ could be simulated.

In the present discussion we assume, in accordance with the paper of Ilie and Equer, ${ }^{23}$ that the effective tunneling masses (TAT, RM) are lower than the extended state effective masses and are equal to $0.01 m_{e}$. A higher, more pessimistic, value of the $\mu c$-Si mobility gap was chosen: $1.54 \mathrm{eV}$ (the conditions for growing quality, thin $\mu c$-Si layers on $a$-Si are rarely met). The parameters outside the TRJ structure were not altered, because they were already selected to fit experimental results. ${ }^{17}$ However, the inclusion of the fielddependent mobility physics called for reoptimization of the TRJ intrinsic layer thickness.

Table III compares the performance of the tandem solar cell with respect to parameter $F_{0}$ for the five different TRJ structures analyzed in Sec. III C. Some interesting conclusions can be extracted from our results. First, the highest efficiencies were obtained with TRJs made entirely of $\mu c$ $\mathrm{Si}$. The efficiency of those tandem solar cells saturates for values of $F_{0}$ lower than $10^{5} \mathrm{~V} / \mathrm{cm}$. This can be visualized for the $n-p(\mu c-\mathrm{Si})$ TRJ in Fig. 10. For low values of $F_{0}$, free carriers can easily diffuse to the region where they recombine. Tandem solar cell efficiency is a function of $F_{0}$ mainly through $F F$, because $J_{\mathrm{SC}}$ and $V_{\mathrm{OC}}$ change very little with respect to $F_{0}$. The optimum intrinsic layer thickness is obviously independent of the presence, absence, or defect density of the intrinsic $\mu c$-Si layer for low values of $F_{0}$. From previous studies made on $a$-Si tandem solar cells with $\mathrm{ST}_{\mu c} n-i-p$ TRJ-looking at the experimental efficiencies achieved $^{18}$ and taking into account Fig. 10-we can only say that the value of the parameter $F_{0}$ should be lower than 5 $\times 10^{5} \mathrm{~V} / \mathrm{cm}$. The dependence of the tandem solar cell efficiency on $F_{0}$ can be neglected only for very high values of $F_{0}\left(>10^{6} \mathrm{~V} / \mathrm{cm}\right)$. Highest solar cell efficiencies are then obtained using thin TRJs, where electric fields are stronger and the electron barrier is therefore thinner. If $n-p$ and $n-i-p$ $(\mu c-\mathrm{Si})$ TRJs are described with very low values of $F_{0}$ $\left(<10^{5} \mathrm{~V} / \mathrm{cm}\right)$, the same tandem solar cell efficiencies are obtained for both types of junctions; the top and bottom $p-i-n$ subcells start to limit the carrier supply, and TRJs no longer influence solar cell performance.

In the $n-p^{+}-p(\mu c-\mathrm{Si} / a-\mathrm{Si} / a-\mathrm{SiC}) \mathrm{TRJ}$ structure, materials of quite different electrical properties are combined. The

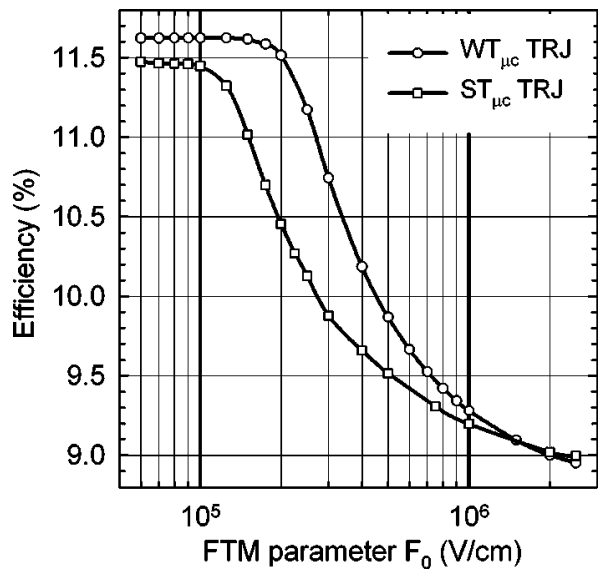

FIG. 10. Dependence of $p-i-n-p-i-n(a-\mathrm{Si} / a-\mathrm{Si} / \mu c-\mathrm{Si} / \mu c-\mathrm{Si} / a-\mathrm{Si} / a-\mathrm{Si})$ tandem solar cell efficiency on the value of the parameter $F_{0}(\mathrm{~V} / \mathrm{cm})$ adopted in the $n-p \mu c$-Si TRJ structure. Simulation results are shown for TRJs with $\mathrm{WT}_{\mu c}$ and $\mathrm{ST}_{\mu c}$ layers. 


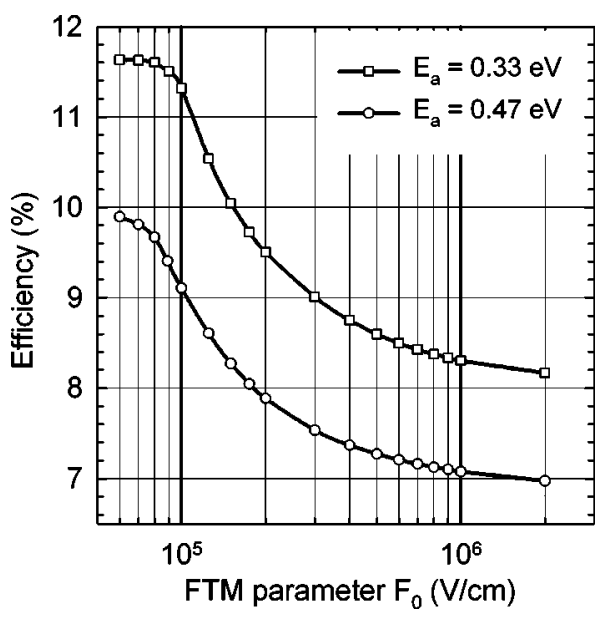

FIG. 11. Dependence of $a-\mathrm{Si} / a$-Si tandem solar cell efficiency on the value of the parameter $F_{0}(\mathrm{~V} / \mathrm{cm})$ adopted in the $n-p^{+}-p(\mu c-\mathrm{Si} / a-\mathrm{Si} / a-\mathrm{SiC})$ TRJ. Simulation results are shown for $p$-type $(a-\mathrm{SiC})$ layer activation energies of $0.33 \mathrm{eV}$ and $0.47 \mathrm{eV}$.

activation energy in doped $\mu c$-Si is much lower than in doped $a$-SiC. The insertion of a highly $p$-doped $a$-Si intermediate layer increases the amount of good recombination. However, this TRJ does not perform as well as the fully $\mu c$-Si TRJ. The experimentally obtained activation energy of the $(a-\mathrm{SiC}) p$ layer of the top $p-i-n$ subcell was $0.47 \mathrm{eV}$. Using the same $p$ layer in the bottom $p-i-n$ led to quite poor solar cell performance. We ran simulations assuming that the activation energy of the bottom subcell $p$ layer was either $0.47 \mathrm{eV}$ or $0.33 \mathrm{eV}$. Table III and Fig. 11 show significant differences obtained in the solar cell efficiencies. All the parameters $\left(V_{\mathrm{OC}}, F F\right.$, and $\left.J_{\mathrm{SC}}\right)$ are lower in the tandem solar cell having the higher activation energy in the $p$ layer of the bottom $p-i-n$ subcell. The high activation energy of the $a$-SiC layer led to poor efficiencies of the $a$-Si tandem cells; moreover, we were not able to reach the $10 \%$ efficiency mark even for very low values of $F_{0}$. High activation energies can be tolerated in the top $p-i-n p$ layer because the front contact can sustain the electric field in the intrinsic layer, and heavily doped $p$ layers are more appropriate for the bottom $p-i-n$ cell.

Table III summarizes the conversion efficiency of $a$-Si/ $a$-Si tandem solar cell with different TRJ structures and leads to some additional observations: the $a$-Si tandem solar cell performance is even worse when no $\mu c$-Si layers are present in the TRJ structure; there is a clear correspondence between higher forward dark currents in the TRJs and higher efficiencies of $a-\mathrm{Si} / a-\mathrm{Si}$ tandem cells using these TRJs.

Our results indicate that only when at least one of the TRJ layers is made of $\mu c-\mathrm{Si}$ can we achieve the reported $a$-Si/ $a$-Si tandem solar cell efficiencies (above $9 \%$ ) for realistic values of $F_{0}$ (around $10^{5} \mathrm{~V} / \mathrm{cm}$ ). If lower mobility gaps for $a-\mathrm{Si}$ and $a-\mathrm{SiC}$ are used in the simulation, then higher values of $F_{0}$ are sufficient for obtaining realistic simulation results. However, $F_{0}$ should be associated with the tunneling current ascribed to the recombination term and is therefore case related and not a fixed value. ${ }^{12}$

\section{TEMPERATURE DEPENDENCE OF J-V TRJ CHARACTERISTICS}

When the temperature dependence of the simulated $J-V$ curves in the range from 200 to $400 \mathrm{~K}$ is compared to experimental results, a significant discrepancy can be observed. Hegedus et al. ${ }^{4}$ published the temperature dependence of the total current at a reverse voltage of $-0.1 \mathrm{~V}$. It was shown that in the $200-400 \mathrm{~K}$ temperature range the current changes by less than one order of magnitude. Assuming that electron and hole mobilities are field dependent, i.e., $\mu(F)$ $=\mu_{0} \exp \left(F / F_{0}\right)$, we obtained in our simulations that for an external voltage of $-0.1 \mathrm{~V}$ the current changes by more than nine orders of magnitude, since the total current is controlled by diffusion, and this mechanism is highly temperature dependent. In order to reduce this dependence, we decided to assume that the field-enhanced free-carrier mobilities are also highly temperature dependent. Following the work published by Juska et al. ${ }^{24}$ we adopted the more general dependence of both mobilities with respect to the electric field and to temperature as: $\mu(F)=\mu_{0} \exp \left(q A F / k T_{F}\right)$, where $A$ is an input parameter expressed in nanometer and $T_{F}$ is given by the expression: $1 / k T_{F}=1 / k T-1 / k T_{\mathrm{CR}} \cdot T_{\mathrm{CR}}$ is a second input parameter (temperature expressed in Kelvin). The expression is known as Gill's law. It specifies an activation energy for the mobility and tends to decrease the dependence of the diffusion current with respect to temperature. The form $\exp (C / k T)$, where $C=q A F$, introduced above, counterbalances the temperature dependence expressed by the form $\exp (-D / k T)$, where $D=E_{C}-E_{F}$ or $D=E_{F}-E_{V}$, characteristic

TABLE IV. Efficiency of $a$-Si/ $a$-Si tandem solar cells with regard to TRJ type and parameter $\left(A, T_{\mathrm{CR}}\right)$ values assume Gill's law was applied for the calculation of charge carrier mobilities. Refer to Table III for the TRJ description.

\begin{tabular}{|c|c|c|c|c|c|}
\hline \multirow[b]{2}{*}{$A(\mathrm{~nm}) / T_{\mathrm{CR}}(\mathrm{K})$} & \multicolumn{5}{|c|}{ Tandem solar cell efficiency (\%) with respect to the TRJ used } \\
\hline & $n-p\left(\mathrm{ST}_{\mu c}\right)^{\mathrm{a}}$ & $n-i-p\left(\mathrm{ST}_{\mu c}\right)^{\mathrm{a}}$ & $n-p^{+}-p\left(E E_{a}\right)^{\mathrm{b}}$ & $n-p^{+}-p\left(L E_{a}\right)^{\mathrm{c}}$ & $n-p\left(\mathrm{LE}_{\mathrm{a}}\right)^{\mathrm{c}}$ \\
\hline $11 / 450$ & - & - & - & - & 7.73 \\
\hline $10 / 450$ & - & - & - & 8.60 & 7.46 \\
\hline $8 / 500$ & 11.59 & 11.55 & 11.60 & 8.51 & 7.36 \\
\hline $8 / 450$ & 11.57 & 11.54 & 11.40 & 8.16 & 7.01 \\
\hline $8 / 400$ & 11.37 & 11.34 & 10.44 & 7.71 & 6.64 \\
\hline
\end{tabular}


of free-carrier concentrations. The computer-predicted temperature dependence of the $J-V$ curves can be adjusted by changing the values of parameters $A$ and $T_{\mathrm{CR}}$.

Adequate values reduce temperature dependence of the total current within the $200-400 \mathrm{~K}$ range to only one order, which agrees quite well with the temperature dependence published by Hegedus et al. The most appropriate parameter values were determined as $T_{\mathrm{CR}}=450 \mathrm{~K}$ and $A=11 \mathrm{~nm}$ in the $n-p \quad(a-\mathrm{Si} / a-\mathrm{SiC}) \quad \mathrm{TRJ}, \quad A=10 \mathrm{~nm}$ in the $n-p^{+}-p \quad(a-$ $\mathrm{Si} / a-\mathrm{Si} / a-\mathrm{SiC}) \mathrm{TRJ}$, and $A=8 \mathrm{~nm}$ in the $n-p^{+}-p$ ( $\mu c-$ $\mathrm{Si} / a-\mathrm{Si} / a-\mathrm{SiC}$ ) TRJ. Table IV shows how different values of parameters $A$ and $T_{\mathrm{CR}}$ affect the performance of $a-\mathrm{Si} / a-\mathrm{Si}$ tandem solar cells. Juska et al. ${ }^{24}$ proposed values of $A$ $=1.6 \mathrm{~nm}$ and $T_{\mathrm{CR}}=430 \mathrm{~K}$ in order to fit the temperature dependence of the measured drift mobility. Alternative equations proposed in Ref. 24 were also tested, but did not improve simulation results.

Table IV does not include simulation results for values of $A$ higher than the ones which match the current levels measured experimentally in the last three TRJs. A straightforward correlation between parameter $F_{0}$ introduced with the FTM model and Gill's law exists, i.e., $F_{0}=k T_{F} / q A$. Therefore, Gill's law can easily be implemented in numerical simulators. It reduces the temperature dependence of the FTM model, making it more appropriate for single TRJ, tandem, and triple solar cell simulations.

\section{CONCLUSIONS}

The numerical simulators ASPIN and D-AMPS were designed for solving the standard semiconductor equation set. Different development paths were chosen. Nevertheless, the simulation results must always meet actual measurements, and this gives us the unique opportunity to study the phenomena with different tools, and above all, it helps us to objectively evaluate our work.

The integration of trap-assisted theories based merely on improved generation-recombination rates into numerical simulators built around the drift-diffusion model undoubtedly requires additional modeling of the tunneling currents. One of the most convenient ways is to include these currents as an addition to the extended state mobility.

Simulation results showed, in agreement with the experimental results, that TRJ tunneling junctions incorporating at least one $\mu c-\mathrm{Si}$ are superior to completely $a-\mathrm{Si}$ structures. Realistic performances of $a$-Si $/ a$-Si tandem solar cells can be simulated assuming that the extended state mobility increases exponentially with the electric field.

A careful analysis of the temperature dependence of the current-voltage characteristics of single recombination junctions indicates that field-enhanced mobility decreases exponentially with the temperature. Proper application of Gill's law, which can be readily implemented in numerical simulators, reduces simulated TRJ current temperature dependence, making it resemble experimental observations. A revised field-enhanced mobility model in conjunction with the trapassisted tunneling model enables reasonable predictions of $a$-Si $/ a$-Si tandem solar cell efficiencies. Nevertheless, further work is recommended to refine the expressions describing mobility with respect to temperature and to electric field strength.

\section{ACKNOWLEDGMENT}

The financial support (Contract No. "Solar Cells" ES/ PA/00-EXI/04) of the Ministry of Education, Science and Sport of the Republic of Slovenia and Secretaria de Ciencia, Tecnologia e Innovacion productiva (SeCyT) is gratefully acknowledged.

${ }^{1}$ F. Smole, Ph. D. thesis, Ljubljana, 1989.

${ }^{2}$ F. A. Rubinelli and R. E. Schropp, Proceedings of the Second World Conference on Photovoltaic Energy Conversion held at Vienna, Austria, 1998, edited by J. Schmid et al.(European Commission, Luxembourg, 1998), pp.998-1001.

${ }^{3}$ P. J. McElheny, J. K. Arch, H. S. Lin, and S. J. Fonash, J. Appl. Phys. 64, 1254 (1988).

${ }^{4}$ S. S. Hegedus, F. Kampas, and J. Xi, Appl. Phys. Lett. 67, 813 (1995).

${ }^{5}$ J. A. Willemen, M. Zeman, J. W. Metselaar, Proceedings of the First World Conference on Photovoltaic Energy Conversion, Waikoloa, Hawaii, 1994 (IEEE, New York, 1995), pp. 599-602.

${ }^{6}$ M. Vukadinović, F. Smole, M. Topič, J. Krč, and J. Furlan, Sol. Energy Mater. Sol. Cells 66, 361 (2001).

${ }^{7}$ J. L. Hartke, J. Appl. Phys. 39, 4871 (1968).

${ }^{8}$ G. Vincent, A. Chantre, and D. Bois, J. Appl. Phys. 50, 5484 (1979).

${ }^{9}$ G. A. M. Hurkx, D. B. M. Klassen, and M. P. G. Knuvers, IEEE Trans. Electron Devices ED-39, 331 (1992).

${ }^{10}$ J. Furlan, Prog. Quantum Electron. 25, 56 (2001).

${ }^{11}$ J. A. Willemen, Ph. D. thesis, Tecnische Universiteit Delft, 1998.

${ }^{12} \mathrm{M}$. Vukadinović, Trap-Assisted Tunneling in Structures of Amorphous and Microcrystalline Silicon (Založba FE in FRI, Ljubljana, 2003).

${ }^{13} \mathrm{~S}$. Selberherr, Analysis and Simulation of Semiconductor Devices (Springer, Vienna, 1984).

${ }^{14}$ M. S. Mock, Analysis of Mathematical Models of Semiconductor Devices (Boole Press Limited, Dublin, 1983).

${ }^{15}$ J. Krč, F. Smole, and M. Topič, Informacije MIDEM 32/1 (Society for Microelectronic Components and Materials (MIDEM), Ljubljana, Slovenia,2002), pp. 6-13.

${ }^{16}$ J. Hou, Ph. D. thesis, The Pennsylvania State University, 1993.

${ }^{17}$ J. K. Rath, F. A. Rubinelli, and R. E. I. Schropp, Proceedings of the Second World Conference on Photovoltaic Energy Conversion held at Vienna, Austria, Ref. ${ }^{2}$, pp. 812-815.

${ }^{18}$ F. A. Rubinelli, J. K. Rath, and R. E. I. Schropp, J. Appl. Phys. 89, 4010 (2001).

${ }^{19}$ F. A. Rubinelli, J. Arch, and S. J. Fonash, J. Appl. Phys. 72, 1621 (1992).

${ }^{20}$ Q. Gu and E. A. Schiff, Phys. Rev. B 52, 5695 (1995).

${ }^{21}$ G. Juška, K. Arlauskas, B. Equer, R. Vanderhaghen, J. Non-Cryst. Solids 227-230, 176 (1998).

${ }^{22}$ H. Antoniadis and E. A. Schiff, Phys. Rev. B 43, 13957 (1991).

${ }^{23}$ A. Ilie and B. Equer, Phys. Rev. B 57, 15349 (1998).

${ }^{24}$ G. Juska, K. Arlauskas, and J. Kocka, J. Non-Cryst. Solids 198-200, 202 (1996). 